\title{
Nonequilibrium scenarios in cluster-forming quantum lattice models
}

\author{
Adriano Angelone, ${ }^{1,2}$ Tao Ying, ${ }^{3}$ Fabio Mezzacapo, ${ }^{4}$ Guido Masella, ${ }^{5}$ Marcello Dalmonte, ${ }^{1,2}$ and Guido Pupillo ${ }^{5}$ \\ ${ }^{1}$ Abdus Salam ICTP, Strada Costiera 11, I-34151 Trieste, Italy \\ ${ }^{2}$ SISSA, Via Bonomea 265, I-34136 Trieste, Italy \\ ${ }^{3}$ Department of Physics, Harbin Institute of Technology, 150001 Harbin, China \\ ${ }^{4}$ Univ Lyon, ENS de Lyon, Univ Claude Bernard, \\ CNRS, Laboratoire de Physique, F-69342 Lyon, France \\ ${ }^{5}$ icFRC and ISIS (UMR 7006), Université de Strasbourg and CNRS, 67000 Strasbourg, France
}

(Dated: June 16, 2020)

\begin{abstract}
We investigate the out-of-equilibrium physics of monodisperse bosonic ensembles on a square lattice. The effective Hamiltonian description of these systems is given in terms of an extended Hubbard model with cluster-forming interactions relevant to experimental realizations with cold Rydberg-dressed atoms. The ground state of the model, recently investigated in Phys. Rev. Lett. 123, 045301 (2019), features, aside from a superfluid and a stripe crystalline phase occurring at small and large interaction strength $V$, respectively, a rare first-order transition between an isotropic and an anisotropic stripe supersolid at intermediate $V$. By means of quantum Monte Carlo calculations we show that the equilibrium crystal may be turned into a glass by simulated temperature quenches and that out-of-equilibrium isotropic (super)solid states may emerge also when their equilibrium counterparts are anisotropic. These out-of-equilibrium states are of experimental interest, their excess energy with respect to the ground state being within the energy window typically accessed in cold atom experiments. We find, after quenching, no evidence of coexistence between superfluid and glassy behavior. Such an absence of superglassiness is qualitatively explained.
\end{abstract}

\section{INTRODUCTION}

The search for ordered or disordered exotic states of matter is a very active field of investigation in condensed matter physics [1 3$]$. The interactions between the individual constituents of a given system play a fundamental role in this context, being intrinsically related to the physical mechanisms responsible for the stabilization of different (possibly novel) physical scenarios. Usually, intriguing equilibrium or out-of-equilibrium (OOE) properties emerge in the presence of frustration, i.e., the impossibility of simultaneously satisfying a minimum energy condition for all terms of the Hamiltonian (see, e.g., Refs. [4, 5]). The latter may arise from, e.g., competing interactions, the presence of peculiar substrates (i.e., lattices) or polidispersity, i.e., the presence in the system of particles with different properties such as, for example, mass and/or size.

Recently, a large class of purely repulsive, isotropic extended-range interactions (ERI), whose relevance ranges from classical soft-matter systems [6 6 ] Rydberg-atom experiments [9 15], has elicited considerable theoretical interest. Indeed, these potentials offer the possibility to explore a variety of equilibrium and OOE phenomena in realistic models where frustration, in the forms discussed above, is not included. The main features of pairwise ERI are a plateau which extends up to interparticle distances of the order of the critical radius $r_{c}$ and a tail quickly approaching zero for $r>r_{c}$ [see Fig. 11(a)]. Systems with ERI at high enough particle density $\rho$ are characterized, in the classical limit, by a so-called cluster crystalline ground state (GS) where crystalline sites are occupied by self-assembled aggregates of particles (i.e., clusters). Classical cluster crystals have been shown to possess peculiar equilibrium dynamical properties resembling those of glass-forming liquids, while still retaining structural order [16]. For these systems, OOE glassy scenarios where disorder coexists with clusterization have also been predicted [17, 18]. Furthermore, when quantum effects are taken into account, clusterization may lead to anomalous Luttinger-liquid behavior in one spatial dimension (1D) $19-22]$, as well as to the coexistence of diagonal long range order and superfluidity (i.e., supersolidity) in 2D free space [23 26] or on a triangular lattice [27]. In the latter, superfluidity may also be concomitant to glassiness in a so-called OOE superglass.

In order to gain theoretical insight into the novel physical phenomena related to clusterization, as well as into its interplay with quantum effects, system geometry, and interaction radius $r_{c}$, it is of crucial interest to extend the investigation to different lattices and choices of relevant parameters. In this context, a recent work by some of us 28] has been devoted to the study of the GS phase diagram of a cluster-forming model of hard-core bosons with shoulder ERI on a square lattice. For such a model the GS is a superfluid (stripe crystal) for sufficiently small (large) interaction strength $V$. Surprisingly, for intermediate values of $V$ a first-order phase transition occurs between two different supersolids: an isotropic one, emerging from the superfluid when $V$ is increased, and an anisotropic stripe supersolid emerging from the partial quantum melting of the large- $V$, essentially classical, crystal.

The study of the GS phases mentioned above required extensive calculations and careful temperature and interaction annealings due to the presence of many OOE states close in energy to the GS. Indeed, it is known that 


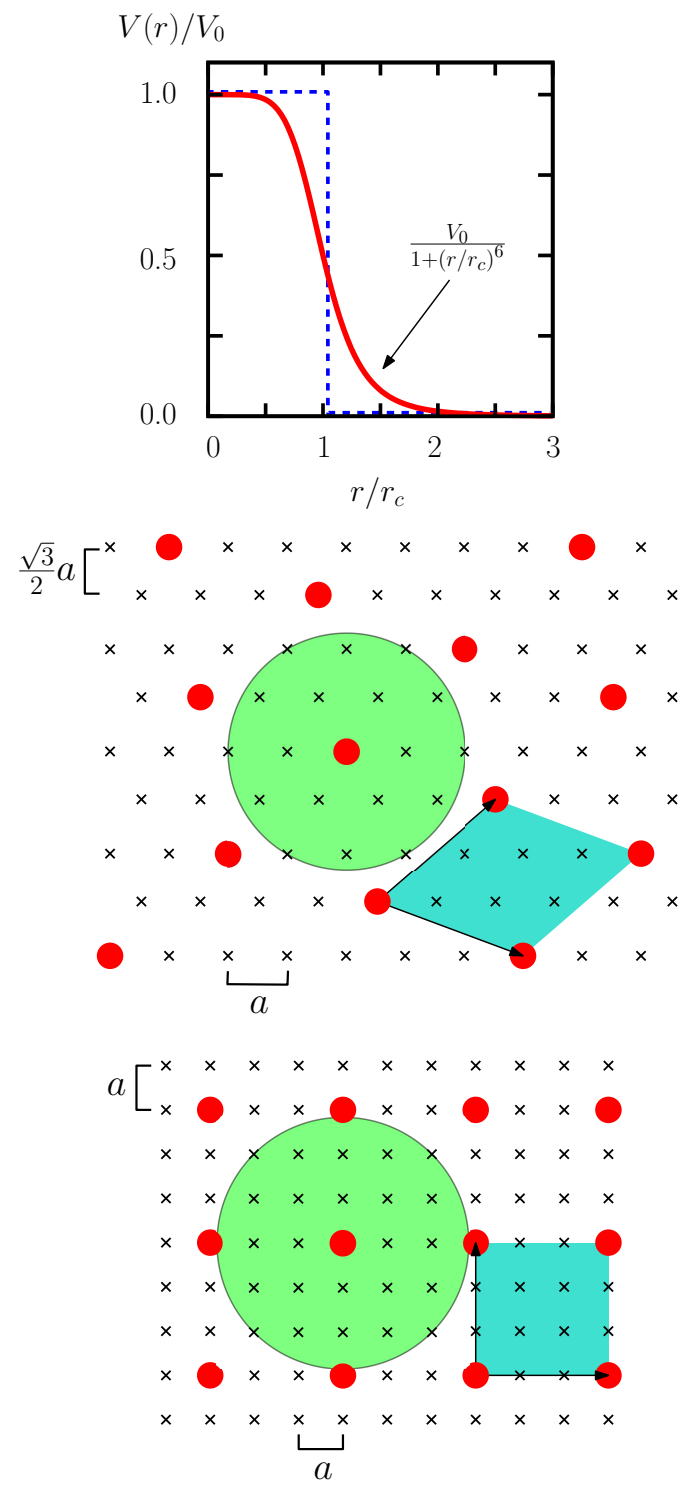

(a)

FIG. 1. Panel (a): examples of extended-range interactions. The continuous and dashed curves represent a soft-shoulder potential (see label) with fast-decaying [i.e., $\left(r / r_{c}\right)^{-6}$ ] tail and a shoulder interaction of strength $V_{0}$ and radius $r_{c}$, respectively. Single-particle crystalline ground states of the extended Bose-Hubbard Hamiltonian in Eq. (1) on a triangular lattice for $r_{c}=2 a$ and $\rho=1 / 7$ [panel (b)], and on a square lattice for $r_{c}=2 \sqrt{2} a$ and $\rho=1 / 9$ [panel (c)]. In panels (b) and (c), black crosses are lattice sites and red dots are occupied sites. Green circles highlight the range of the interaction around an occupied site, black arrows are the vectors generating the crystalline structure, and cyan regions indicate the primitive cells of the crystals.

extended-range interactions on a lattice lead to a plethora of low-energy metastable states, whose number exponentially increases with the system size [29, 30]. Such OOE states are of importance to possible quantum simulations of our model of interest with cold atoms since in these experiments states whose energy is above the GS one are commonly accessed (even when one is interested in GS physics, due in this case to the presence of undesired excitations). As an example, even in the case of the preparation of a bosonic Mott insulator (a comparatively simpler state with respect to the ones discussed in this work) one can currently obtain up to defect densities of up to the percent level, which (in the strongly interacting regime) correspond to differences in energy density of up to a few percent. As a consequence, the fundamental question which need be addressed is whether or not theoretical predictions that one can make for the GS are stable within the characteristic energy window accessible to experiments [31].

In this work the OOE scenarios of the model studied in 28] are systematically investigated by means of a path integral Monte Carlo (PIMC) approach. In particular, our system can be driven out of equilibrium via PIMC low-temperature $(T)$ quenches.

Our main findings are the following. (i) As opposed to the isotropic and anisotropic supersolid GSs, a lowtemperature quench leads to largely isotropic, OOE (super)solid states. Remarkably, these are also found for values of $V$ at which the equilibrium phases are instead anisotropic. (ii) Similarly to our previous study of the same (albeit with different $r_{c}$ and $\rho$ ) quantum model on the triangular lattice [27], as well as to that of the classical model in free space [17], the OOE counterpart of the equilibrium crystal at large $V$ is a normal glass. (iii) In the investigated parameter range no evidence of superglassy behavior is obtained. The occurrence of such a state, which has been predicted for the triangular lattice, crucially depends on the interplay between lattice geometry, particle density, and interparticle interactions.

It is worth mentioning that the energy deviations from the GS of the OOE states analyzed in this work are comparable to those routinely obtained in cold atom experiments.

The remainder of this paper is organized as follows. In the next section we describe the details of the Hamiltonian model of our interest with particular attention to its cluster-forming regimes and, briefly, the numerical method adopted to carry out our investigation. In Sec. III we present and discuss our results, while in the last section we outline the conclusions of our work.

\section{MODEL AND METHODS}

The model we investigate is described by the Hamiltonian

$$
H=-t \sum_{\langle i j\rangle}\left(b_{i}^{\dagger} b_{j}+\text { h.c. }\right)+V \sum_{i<j: r_{i j} \leq r_{c}} n_{i} n_{j}
$$

on a square lattice of $N=L \times L$ sites and lattice constant $a$ with periodic boundary conditions. Here $t$ is the hopping coefficient between nearest-neighbor sites, $b_{i}$ 
and $b_{i}^{\dagger}$ are annihilation and creation operators for hardcore bosons on site $i$, respectively, $n_{i}=b_{i}^{\dagger} b_{i}, V$ is the interaction strength, and $r_{i j}$ is the distance between sites $i$ and $j$. In the following, $a$ and $t$ will be taken as units of length and energy, respectively.

For $r_{c}=a$, i.e., nearest-neighbor potential, the phase diagram of the model contains superfluid, solid, and insulating phases [32], while supersolid states can be stabilized adding longer-ranged density-density interactions [33, 34]. We study the model for $r_{c}>a$, in a regime where cluster formation takes place in the system.

For low enough $\rho$, the classical (i.e., $t=0$ ) GS is a zero-energy single-particle crystal, where the interparticle spacing is larger than the interaction radius. The maximum density $\rho_{c}$ for which such a crystal exists is determined by $r_{c}$ and the lattice geometry. For example, in our study of Eq. (11) on the triangular lattice the choice $r_{c}=2 a$ results in a critical density $\rho_{c}^{\mathrm{tr}}=1 / 7$. In the equilibrium study performed in [28], on the other hand, a square lattice geometry with $r_{c}=2 \sqrt{2} a$ leads to a critical density $\rho_{c}^{\mathrm{sq}}=1 / 9$. The single-particle crystalline structures corresponding to these densities are shown in Figs. 1(b) and 1(c), respectively.

For $\rho \gtrsim \rho_{c}$, a single-particle solid has a higher potential energy than a solid in which particles group up in tightly packed clusters. Indeed, the latter can arrange themselves far enough from each other to be noninteracting (i.e., outside of their mutual interaction radius). A larger value of $\rho$ results in the formation of larger clusters. For instance, on the triangular lattice the chosen value of $\rho=13 / 36 \sim 2.5 \rho_{c}^{\text {tr }}$ led to clusters of three to four particles on average, while on the square lattice, for $\rho=5 / 36 \sim 1.25 \rho_{c}^{\mathrm{sq}}$, the largest clusters contain two particles.

When the system is driven away from thermal equilibrium, cluster formation can cause effective polidispersity, which in turn plays a fundamental role in the appearance of (super)glassy states [17, 27]. This phenomenon is favored by large cluster sizes, as well as (sufficiently) strong interactions, which prevent particles from delocalizing between different clusters, and entire clusters from spatially rearranging to establish an ordered (crystalline) structure.

In this work we analyze the OOE physics of Eq. (1) for the same parameter range investigated in [28]. The model shows a rich GS phase diagram, characterized by, e.g., competing supersolid phases. Understanding the robustness of these scenarios within the typical experimental accuracy is one of the main objectives of the present study. Furthermore here, due to the presence of significantly smaller clusters, frustration effects should be significantly weaker than those occurring in the study of Ref. [27] on the triangular lattice. This would allow one to determine, for instance, to which degree various OOE phenomena depend on clusterization.

We study the model Eq. (11) by means of path integral quantum Monte Carlo simulations using Worm updates [35]. This is a state-of-the-art technique, which yields numerically exact results for unfrustrated bosonic systems and allows one to accurately estimate observables such as the superfluid fraction $\rho_{s} / \rho=$ $(4 \beta t \rho)^{-1}\left\langle W_{x}^{2}+W_{y}^{2}\right\rangle$ and the static structure factor $S(\mathbf{k})=N^{-2} \sum_{i j} \exp \left[-i \mathbf{k}\left(\mathbf{r}_{i}-\mathbf{r}_{j}\right)\right]\left\langle n_{i} n_{j}\right\rangle$. These order parameters measure superfluidity and crystalline order, respectively, and are defined in terms of the inverse temperature $\beta=\left(k_{B} T\right)^{-1}\left(k_{B}\right.$ is the Boltzmann constant, set to one in the following), of the winding number $W_{x}, W_{y}$ in direction $x, y$, respectively, and of the lattice wave vectors $\mathbf{k}$. Here, $\langle\ldots\rangle$ stands for statistical average. We also estimate the renormalized EdwardsAnderson parameter $Q_{\mathrm{EA}}=\sum_{i}\left\langle n_{i}-\rho\right\rangle^{2} / Q_{\mathrm{EA}}^{0}$, a wellknown observable which allows one to identify glass behavior in lattice systems in the absence of crystalline order. The normalization $Q_{\mathrm{EA}}^{0}=N \rho(1-\rho)$ is the value obtained for a fully localized state. Finally, we determine the single-particle Green function defined as $G(\mathbf{r})=N^{-1} \sum_{i}\left\langle b_{i}^{\dagger} b_{i+\mathbf{r}}\right\rangle$, associated to the presence of offdiagonal quasi-long-range order in our two-dimensional system.

We perform large-scale simulations with up to $N=$ $96 \times 96$ sites and temperatures between $T / t=1$ and $T / t=1 / 20$, the latter yielding essentially GS results in the equilibrium case 28]. To gain insight into the OOE scenarios, we employ a simulated quench protocol, by running low- $T$ simulations starting from high- $T$ configurations without performing simulated annealing steps in $T$. The experimental relevance (in the sense discussed in Sec. I) of our obtained OOE states is assessed a posteriori. In particular, our estimated OOE energies never exceed the GS ones by more than $3.5 \%$.

\section{RESULTS}

For clarity, we begin our discussion by summarizing the GS phase diagram of model Eq. (11) (we refer the reader to Ref. 28] for an exhaustive discussion). The GS (lower part of Fig. 2) is a superfluid (SF) at weak interactions, which makes way for an isotropic supersolid (IS) at $V / t=2.6$. The system then undergoes a first-order transition at $V / t=4.0$ to a supersolid state with anisotropic stripe crystalline structure and superfluid response, i.e., a stripe supersolid (SS). Finally, superfluidity is lost at $V / t=4.45$, and the GS becomes a stripe crystal $(\mathrm{SC})$.

Driving the system away from thermal equilibrium results in the OOE phase diagram shown in the upper part of Fig. 2, obtained via analysis of the relevant observables shown in Figs. 3 and 4 and discussed below. In the strongly interacting regime, i.e., $V / t>5.5$, and for temperatures $T / t<1 / 5$, the simulated quenches stabilize OOE states where diagonal long range order vanishes in the thermodynamic limit. As signaled by the finite value of $Q_{\mathrm{EA}}$ (see Fig. 3), concomitant to the absence of superfluidity, the resulting states are normal, essentially 


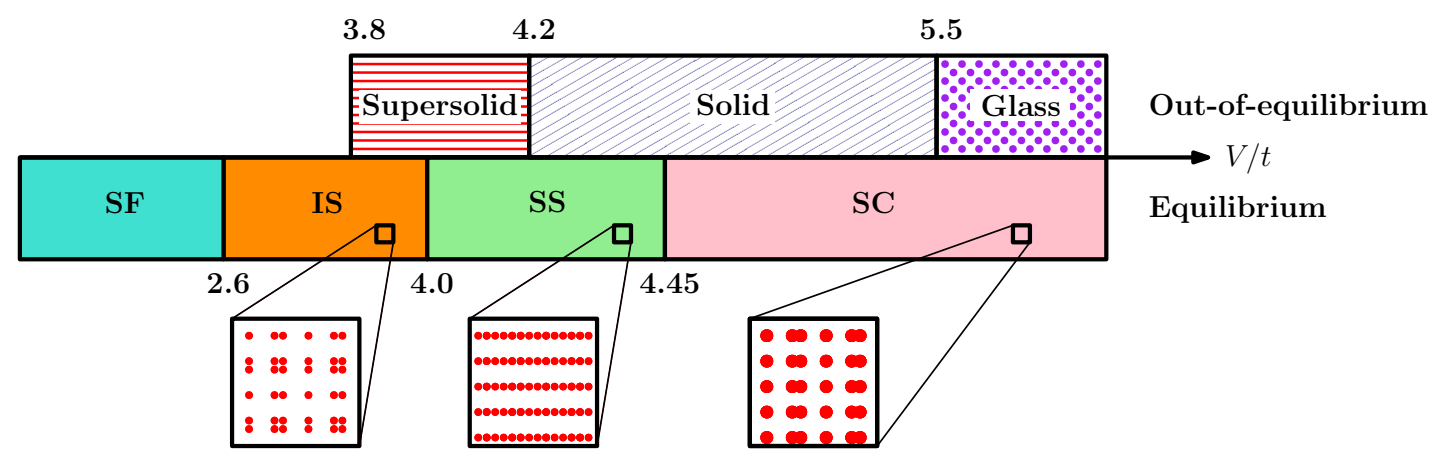

FIG. 2. Schematic phase diagram of Eq. (1) as a function of the interaction strength $V / t$. Each colored region in the lower part of the figure corresponds to a GS equilibrium phase: namely, a superfluid (SF, cyan), an isotropic supersolid (IS, orange), a stripe supersolid (SS, green) and a stripe crystal (SC, pink). The drawings are sketches of the crystalline structure (where present) of each equilibrium phase. The filling patterns in the upper part of the diagram identify the OOE states reached via simulated temperature quenching at target temperature $T / t=1 / 20$. The regions where quenching leads to OOE supersolid, solid and glassy states are denoted by horizontal, diagonal, or dot filling patterns, respectively.

classical, glasses [36].

Conversely, following our quenches at $T / t=1 / 20$ in the intermediate- $V / t$ region (i.e., $3.8 \leq V / t \leq 4.6$ ) the system retains long-range order, reaching OOE states with crystalline structures different from those obtained at equilibrium [see Fig. 4(d)]. These results allow one to identify a variety of crystalline states in the OOE phase diagram, as different realizations of each simulation may converge to states with different strength and type of diagonal long-range order. For $V / t<4.2$ the system displays superfluid behavior [see Fig. 4 (c)]. The latter coexists with diagonal long-range order down to $V / t=3.8$, pointing out the occurrence of OOE supersolid states in this parameter range. For $V / t<3.8$ our quenching process is ineffective, and the system equilibrates to an IS and a $\mathrm{SF}$ for $V / t>2.6$ and $V / t<2.6$, respectively. Remarkably, the OOE supersolids display features considerably different from their equilibrium counterparts. Specifically, both superfluid responses and crystalline order are essentially isotropic even when the corresponding equilibrium supersolids are strongly anisotropic.

In both the high- and intermediate- $V / t$ region, we determine the degree of equilibration of each simulated quench by performing it in several (i.e., $\gtrsim 30$ ) independent realizations, differing in both the initial configuration and in the thermalization seed of the QMC simulation. This procedure mimics what is customarily done in the laboratory, where many realizations of an experiment are performed to measure the observables of interest.

If our quench protocol does not drive the system away from thermal equilibrium, all the realizations converge to the equilibrium state, and the details of the QMC stochastic dynamics in configuration space are inessential. On the other hand, where the OOE driving succeeds, most of the realizations fail to equilibrate, with their initial conditions becoming crucial in determining the state reached by each simulation. A typical example of the latter behavior is shown in Fig. 3(a), where the mean value of an observable (in this case, the maximum value of the structure factor) strongly depends on the realization.

In general, for different realizations of a quench at a chosen parameter set, observables may take different values; however, each realization is found to display the hallmark features of the corresponding OOE phase. For example, in the OOE supersolid region of Fig. 2 some realizations may yield crystalline orders of different strength, as well as different values of superfluid fraction. Nevertheless, each realization displays finite superfluid fraction and concomitant diagonal long-range order.

Figures 3(b)-3(d) show the scaling, as a function of the system size, of the realization-averaged maximum peak of the structure factor $S_{\max }$ and of the Edwards-Anderson parameter $Q_{\mathrm{EA}}$ after quenching to different target temperatures for $V / t=5.0$ and $V / t=6.0$ (filled and empty symbols, respectively). For these values of $V / t$, the observed OOE states are non-superfluid. We find equilibration to a stripe solid for $T / t=1 / 5$ (triangles). A decrease of the target temperature results in failure to equilibrate the vast majority of realizations, which converge to states where diagonal long range order is suppressed with respect to the equilibrium scenario. For $V / t=5.0, S_{\max }$ remains finite in the thermodynamic limit for $T / t=1 / 10$ (filled squares) and $T / t=1 / 20$ (filled circles), signaling an OOE crystal. Conversely, crystalline order is lost for $V / t=6.0$ and $T / t=1 / 10$ (empty squares) and $T / t=1 / 20$ (empty circles). For these temperatures, $Q_{\mathrm{EA}}$ remains finite [panel (d)], signaling the emergence of glassy behavior.

Figure 4 shows a detailed comparison of the realization-averaged superfluid and crystalline order parameters for the OOE and equilibrium cases (empty and filled symbols, respectively) as a function of $V / t$. Supersolid behavior occurs, after quenching, for $3.8 \leq V / t<$ 4.2 , i.e., in an interaction strength window smaller than that for which supersolidity is found at equilibrium. In particular, superfluidity vanishes in the thermodynamic limit for $V / t \geq 4.2$ [diamonds in Fig. 匹(b)]. As men- 


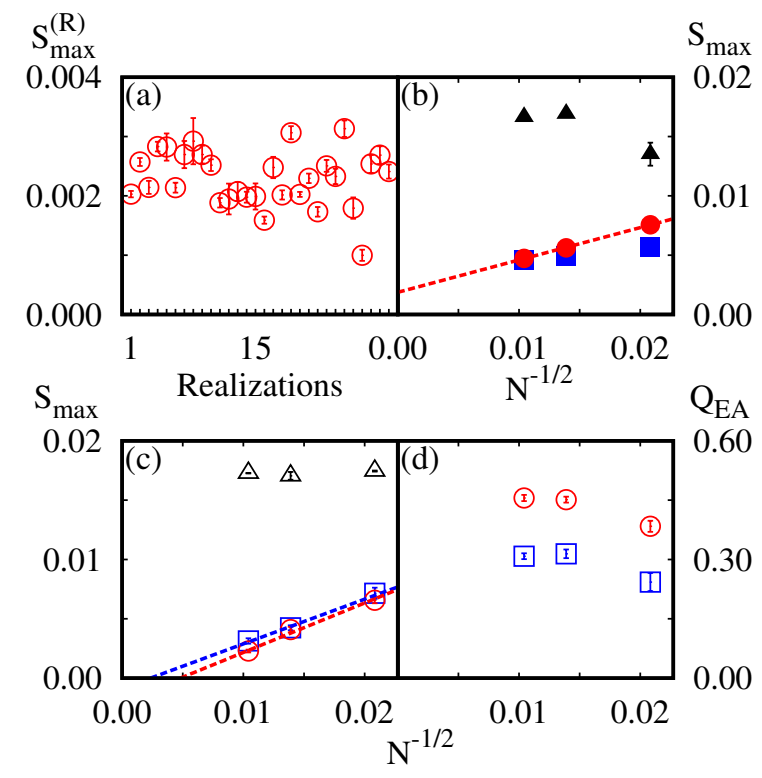

FIG. 3. Results for finite-temperature simulated quenches performed at $V / t=5.0$ and $V / t=6.0$. Panel (a): maximum value of the realization-dependent structure factor $S_{\max }^{(R)}$ as a function of the realization index for $L=96, T / t=1 / 20$, and $V / t=6.0$. Panel (b): realization-averaged value of $S_{\max }$ as a function of the inverse system size for $V / t=5.0$. Panel (c): same as for panel (b) for $V / t=6.0$. Panel (d): realization-averaged Edwards-Anderson parameter $Q_{\mathrm{EA}}$ as a function of the inverse system size for $V / t=6.0$. In all panels, filled (empty) symbols correspond to $V / t=5.0$ $(V / t=6.0)$, while triangles, squares and circles correspond to $T / t=1 / 5,1 / 10,1 / 20$, respectively. The dashed lines correspond to linear fits in $N^{-1 / 2}$, shown when estimates for the two largest sizes are not identical within numerical uncertainty.

tioned above, the details of these supersolid OOE states may be both quantitatively and qualitatively different from the equilibrium ones, whose order parameters are denoted for clarity by $\rho_{s}^{\mathrm{eq}} / \rho$ and $S_{\max }^{\mathrm{eq}}$, respectively. As expected, for small $V / t \lesssim 4$ our quenching protocol does not significantly alter the values of $\rho_{s} / \rho$ and $S_{\max }$ with respect to $\rho_{s}^{\mathrm{eq}} / \rho$ and $S_{\max }^{\mathrm{eq}}$ (Fig. 4(c) and 4(d) and finite-size scaling for $V / t=3.9$ [triangles in panels (a) and (b)]). For intermediate $V / t$, while at the IS/SS transition $S_{\max }^{\text {eq }}$ [filled squares in Fig. [4(d)] develops strong anisotropy [28] and features a sizable variation, $S_{\max }$ remains essentially constant [empty squares in Fig. 4(d)] and isotropic. Indeed, in all quench realizations the maximum peaks of the structure factor occur at realization-dependent wave vectors $\left(k_{x}^{(R)}, 0\right)$ and $\left(0, k_{y}^{(R)}\right)$ with $k_{x}^{(R)} \simeq k_{y}^{(R)}$ and $S\left(k_{x}^{(R)}, 0\right) \simeq S\left(0, k_{y}^{(R)}\right)$. Similarly, $\rho_{s} / \rho$ [empty circles in Fig. 4(c)] takes considerably lower values than $\rho_{s}^{\text {eq }} / \rho$ [filled circles in Fig. 4(c)] for $V / t \gtrsim 4.0$ and the superfluid response, as opposed to what is found at equilibrium, is essentially isotropic. This clarifies the difference between the equilibrium supersolid states, which can be either isotropic or anisotropic, and the OOE ones, which are found to be always largely isotropic. Such a difference persists even in the absence of superfluidity in the OOE states: for example, for $V / t>4.45$ the GS is a stripe crystal while quenching results in the appearance of substantially isotropic crystals and glasses.
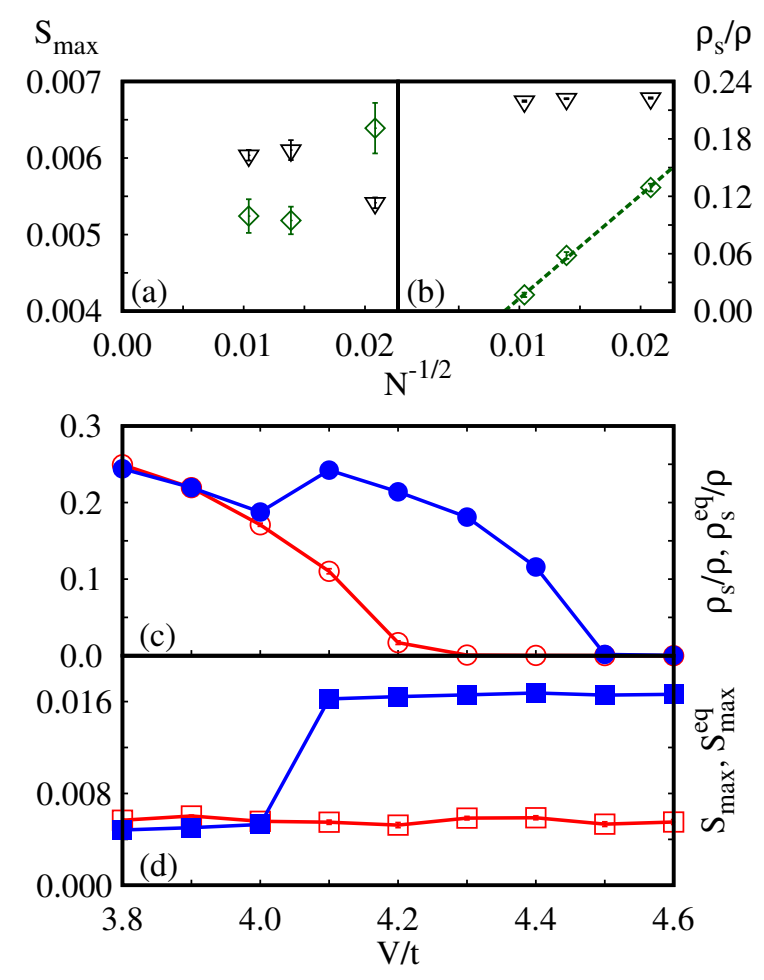

FIG. 4. Panels (a),(b): scaling in the inverse size of $S_{\max }$ and $\rho_{s} / \rho$ for $V / t=3.9$ (triangles) and $V / t=4.2$ (diamonds) at $T / t=1 / 20$. Dashed lines are linear fits to the numerical data, shown when estimates for the two largest sizes are not identical within their uncertainty. Panel (c): comparison between the equilibrium superfluid fraction $\rho_{s}^{\text {eq }} / \rho$ (filled circles) and the OOE one $\rho_{s} / \rho$ (empty circles) as a function of the interaction strength at $T / t=1 / 20$ and $L=96$. Panel (d): comparison of the equilibrium maximum value of the structure factor $S_{\max }^{\mathrm{eq}}$ (filled squares) and the OOE one $S_{\max }$ (empty squares) for the same parameters of panel (c). In panels (c) and (d), solid lines are guides to the eye. In all panels, OOE estimates are realization-averaged.

The isotropic character of the OOE states can also be inferred by inspection of $G_{x}$ and $G_{y}$, i.e., the singleparticle Green function $G(\mathbf{r})$ along the $x$ and $y$ directions, respectively. For $V / t=4.1, G_{x}$ and $G_{y}$ of the corresponding anisotropic SS GS [triangles and circles in Fig. [5(a), respectively] are clearly different. Specifically, while both decay algebraically as a function of the distance, signaling off-diagonal quasi-long-range order, $G_{y}$ is characterized by oscillations in correspondence of the stripe periodicity. The OOE $G(\mathbf{r})$, on the other hand, is essentially isotropic, i.e., $G_{x} \sim G_{y}$ [squares in Fig. [5(a)].

Figure 5(b) displays the same comparison for $V / t=$ 6.0. Here the decay of the $G(\mathbf{r})$, both at equilibrium and 


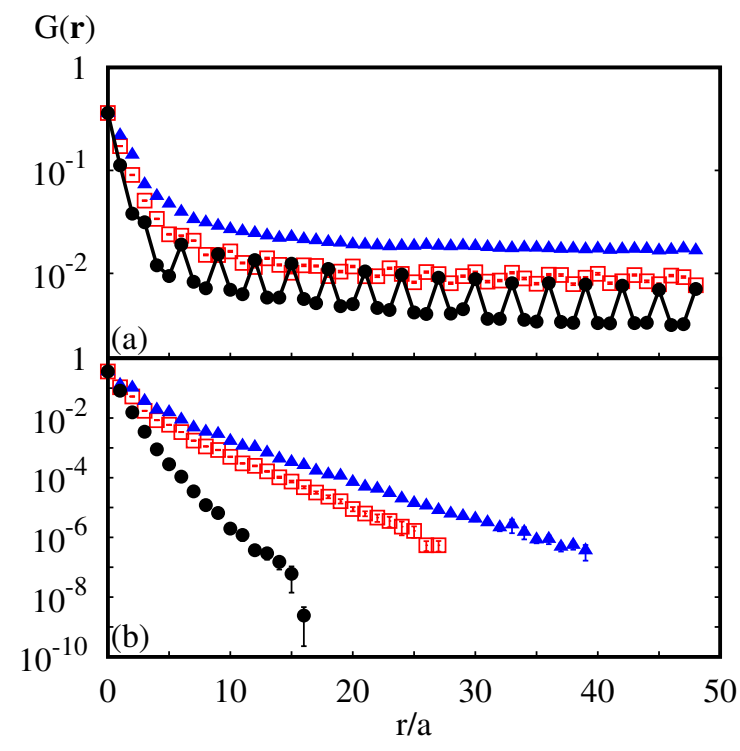

FIG. 5. Panel (a): single-particle Green function $G(\mathbf{r})$ for $T / t=1 / 20, L=96$, and $V / t=4.1$. Triangles and circles refer to the equilibrium $G(\mathbf{r})$ along the $x$ and $y$ direction, respectively (see text), while squares denote the OOE $G(\mathbf{r})$ along the $y$ direction. The corresponding OOE $G(\mathbf{r})$ along the $x$ direction (not shown) is essentially identical. Continuous lines are guides to the eye. Panel (b): same as panel (a) for $V / t=6.0$.

OOE, is exponential, as expected for a nearly classical crystal and a glass, respectively. Also in this case, the equilibrium $G(\mathbf{r})$ is strongly anisotropic, while $G_{x} \sim G_{y}$ in its OOE counterpart.

Further insight into the OOE physics of our model can be gained from the occupation maps in Fig. 6] In both cases shown in the figure $[V / t=6.0$ in panel (a) and $V / t=4.1$ in panel (b)] particles clusterize; for $V / t=6.0$, clusters have in general different shapes and orientations. These induce an effective polidispersity, ultimately resulting in glassy behavior [17]. On the other hand, for $V / t=4.1$, where the system is supersolid, particles can "hop" between different clusters, establishing long exchange cycles which give rise to a sizable superfluid response. The latter is concomitant with a well defined crystalline structure. While different kinds of diagonal long-range order may appear depending on the realization, the vast majority of the latter lead to the same type of crystalline order of the supersolid state shown in Fig. $6(\mathrm{~b})$.

It is important to mention that no evidence of superglassy behavior has been found in the parameter range investigated in this work. This constitutes an important difference with respect to the results of our study of model Eq. (11) for $r_{c}=2$ on the triangular lattice [27]. Qualitative insight into this difference can be obtained by analyzing the two fundamental ingredients of a superglass phase: namely, superfluidity and frustration.

A source of frustration, in our observed OOE states, is

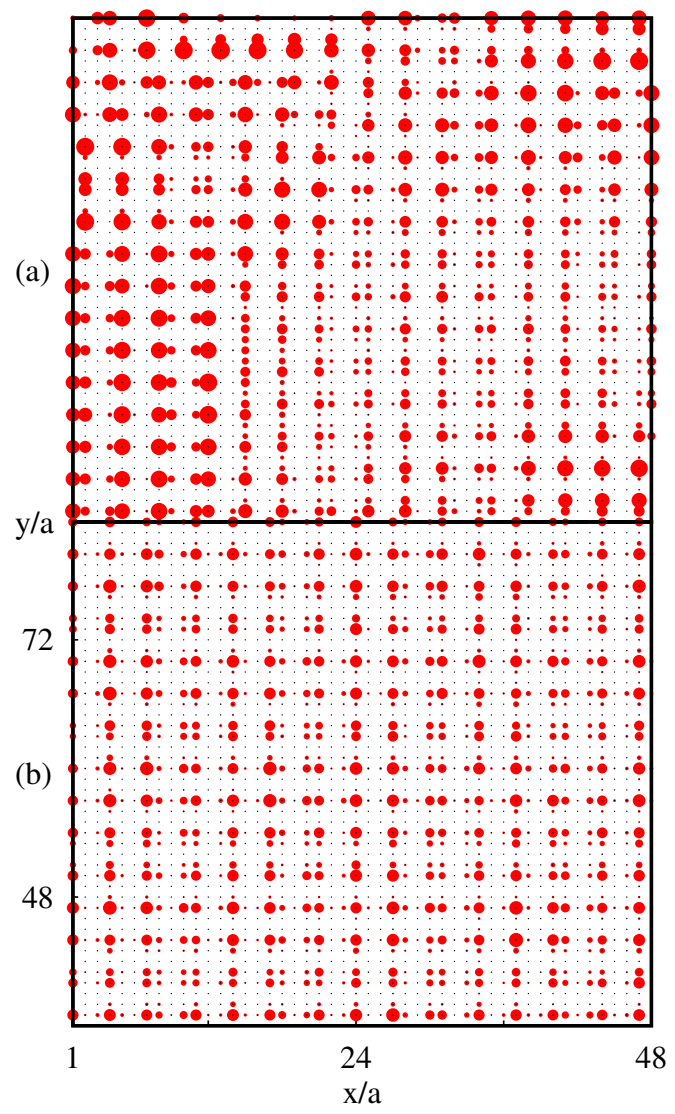

FIG. 6. Panel (a): portion of a site occupation map for one realization for $L=96, T / t=1 / 20$, and $V / t=6.0$. Panel (b): same as panel (a) for $V / t=4.1$. In both panels, black dots correspond to lattice sites, while the size of the red dot on each site is proportional to its occupation.

cluster formation, and the resulting self-induced effective polidispersity [17. In this framework, frustration is magnified for increasing cluster sizes (whose typical value is related to the ratio $\rho / \rho_{c}$, see Sec. II) and/or interaction strength, since cluster formation takes place as a mechanism to decrease the potential energy of the system, and this effect is more important when $V / t$ is large. This allows one to have strong frustration even with very small clusters, but in general may not allow one to obtain a superglass, since for very strong interactions superfluidity is also suppressed, and one obtains only a normal (i.e., insulating) glass.

In our study on the triangular lattice we choose $\rho \sim$ $2.5 \rho_{c}^{\mathrm{tr}}$; as a result, clusters of three to four particles are formed in the obtained OOE states. Superglassy behavior is found because frustration-induced suppression of crystalline order occurs, due to the relatively large cluster size, at moderate values of $V / t$, where a sizable superfluid fraction is found at equilibrium and survives in the OOE scenario. Conversely, on the square lattice we consider $\rho \sim 1.25 \rho_{c}^{\mathrm{sq}}$. As discussed above, this results in smaller clusters and weaker frustration effects at a given $V / t$. In 
order to observe the structural order loss characteristic of a glassy state one therefore needs to go at interaction strengths high enough $(V / t \sim 5.5)$ that superfluidity is suppressed.

A further numerical indicator of the difference between the two systems can be given using a simple energetic argument. Indeed, the ratio $\Lambda$ between potential and kinetic energy can be used to roughly estimate particle mobility. When $\Lambda$ is large, e.g., for large $V / t$ or large $\rho / \rho_{c}$, particles, and clusters formed after quenching, are strongly localized, preventing the realization of a crystalline structure. Superglassy behavior emerges as a delicate balance between localization and superfluidity, which conversely takes place, at low $T / t$, for small $\Lambda$.

Indeed, on the triangular lattice superglasses were observed as the OOE counterparts of supersolids at $\Lambda \sim 9$ and $\rho_{s} / \rho \sim 0.1$. On the other hand, the equilibrium supersolid phases of model Eq. (1) on the square lattice are characterized by much smaller $\Lambda \sim 1.2$ and higher $\rho_{s} / \rho \sim 0.2$. Here the OOE driving leads to superfluid states where, due to larger mobility, crystalline order can be restored, and glassy states can only be obtained at larger $V / t$, where superfluidity is suppressed.

\section{CONCLUSIONS AND OUTLOOK}

We study the out-of-equilibrium scenarios of a model of monodispersed hard-core bosons on a square lattice with an extended-range potential of the shoulder type, of interest for experiments with cold Rydberg-dressed atoms. In the parameter region of our investigation, the ground state of the model is a cluster crystal and a superfluid for strong and weak interactions respectively, and a rare transition between an isotropic and an anisotropic supersolid state occurs for intermediate interaction strength.
Via simulated temperature quenches, we obtain glasses for strong interactions, while for moderate values of the latter (super)solids appear. Such (super)solids are qualitatively different from the ground state ones, being essentially isotropic even for values of the interaction strength for which the corresponding ground state is anisotropic. The out-of-equilibrium states we find display energy deviations with respect to the ground state which are comparable with those of common cold atom experiments.

For all interaction strength values where out-ofequilibrium superfluidity remains finite, long-range crystalline order is also maintained. Therefore, no evidence of superglassy behavior is found in our region of investigation, as opposed to the case of the triangular lattice, where we demonstrated such an exotic state. Indeed, the choice of a smaller particle density on the square lattice leads to frustration effects strong enough to cause the loss of crystalline order only at an interaction value where superfluidity is also lost.

\section{ACKNOWLEDGEMENTS}

We acknowledge useful discussion with A. Kuklov, W. Lechner, M. Mattioli, and S. Wessel. Work in Strasbourg was supported by the grant ANR-ERA-NET QuantERA - Projet RouTe (No. ANR-18-QUAN-0005-01). G. P. acknowledges support from the Institut Universitaire de France (IUF) and USIAS. G. M. was also supported by the French National Research Agency (ANR) through the Programme d'Investissement d'Avenir under contract ANR-17-EURE-0024. T. Y. was also supported by the National Natural Science Foundation of China (Grant No. 11504067). Work in Trieste was supported by the ERC under Grant No. 758329 (AGEnTh) and by the EU Quantum Flagship grant PASQuanS.
[1] M. Boninsegni and N. V. Prokof'ev, Rev. Mod. Phys. 84, 759 (2012)

[2] R. Nandkishore and D. A. Huse, Annual Review of Condensed Matter Physics 6, 15 (2015)

[3] X.-L. Qi and Z. S.-C., Physics Today 63, 33 (2010)

[4] S. F. Edwards and P. W. Anderson, Journal of Physics F: Metal Physics 5, 965 (1975)

[5] K. Binder and A. P. Young, Rev. Mod. Phys. 58, 801 (1986)

[6] B. M. Mladek, D. Gottwald, G. Kahl, M. Neumann, and C. N. Likos, Phys. Rev. Lett. 96, 045701 (2006).

[7] D. A. Lenz, R. Blaak, C. N. Likos, and B. M. Mladek, Phys. Rev. Lett. 109, 228301 (2012).

[8] E. Sciortino, Francesco; Zaccarelli, Nature 493, 30 (2013).

[9] M. Saffman, T. Walker, and K. Mølmer, Rev. Mod. Phys. 82, 2313 (2010)

[10] Y.-Y. Jau, A. M. Hankin, T. Keating, I. H. Deutsch, and G. W. Biedermann, Nature Physics 12, 71 (2015).
[11] J. Zeiher, R. van Bijnen, P. Schauß, S. Hild, J. yoon Choi, T. Pohl, I. Bloch, and C. Gross, Nature Physics 12, 1095 (2016).

[12] T. Lahaye, C. Menotti, L. Santos, M. Lewenstein, and T. Pfau, Rep. Prog. Phys. 72, 126401 (2009) 0905.0386.

[13] R. Löw, H. Weimer, J. Nipper, J. B. Balewski, B. Butscher, H. P. Büchler, and T. Pfau, J. Phys. B 45, 113001 (2012).

[14] H. Bernien, S. Schwartz, A. Keesling, H. Levine, A. Omran, H. Pichler, S. Choi, A. S. Zibrov, M. Endres, M. Greiner, V. Vuletić, and M. D. Lukin, Nature 551, 579 (2017).

[15] S. de Lsleuc, V. Lienhard, P. Scholl, D. Barredo, S. Weber, N. Lang, H. P. Bchler, T. Lahaye, and A. Browaeys, arXiv:1810.13286.

[16] R. Díaz-Méndez, F. Mezzacapo, F. Cinti, W. Lechner, and G. Pupillo, Phys. Rev. E 92, 052307 (2015).

[17] R. Díaz-Méndez, F. Mezzacapo, W. Lechner, F. Cinti, E. Babaev, and G. Pupillo, Phys. Rev. Lett. 118, 067001 (2017) 
[18] R. Diaz-Mendez, G. Pupillo, F. Mezzacapo, M. Wallin, J. Lidmar, and E. Babaev, Soft Matter 15, 355 (2019).

[19] M. Mattioli, M. Dalmonte, W. Lechner, and G. Pupillo, Phys. Rev. Lett. 111, 165302 (2013).

[20] M. Dalmonte, W. Lechner, Z. Cai, M. Mattioli, A. M. Läuchli, and G. Pupillo, Phys. Rev. B 92, 045106 (2015).

[21] M. Motta, E. Vitali, M. Rossi, D. E. Galli, and G. Bertaina, Phys. Rev. A 94, 043627 (2016).

[22] M. Teruzzi, D. E. Galli, and G. Bertaina, Journal of Low Temperature Physics 187, 719 (2017).

[23] N. Henkel, R. Nath, and T. Pohl, Phys. Rev. Lett. 104, 195302 (2010).

[24] N. Henkel, F. Cinti, P. Jain, G. Pupillo, and T. Pohl, Phys. Rev. Lett. 108, 265301 (2012).

[25] F. Cinti, P. Jain, M. Boninsegni, A. Micheli, P. Zoller, and G. Pupillo, Phys. Rev. Lett. 105, 135301 (2010)

[26] F. Cinti, T. Macr, W. Lechner, G. Pupillo, and T. Pohl, Nature Communications 5, 1 (2014)

[27] A. Angelone, F. Mezzacapo, and G. Pupillo, Phys. Rev. Lett. 116, 135303 (2016)

[28] G. Masella, A. Angelone, F. Mezzacapo, G. Pupillo, and N. V. Prokof'ev, Phys. Rev. Lett. 123, 045301 (2019)

[29] C. Menotti, C. Trefzger, and M. Lewenstein, Phys. Rev. Lett. 98, 235301 (2007).

[30] C. Trefzger, C. Menotti, and M. Lewenstein, Phys. Rev. A 78, 043604 (2008)
[31] I. Bloch, J. Dalibard, and S. Nascimbene, Nature Physics 8, 267 (2012).

[32] G. Schmid, S. Todo, M. Troyer, and A. Dorneich, Phys. Rev. Lett. 88, 167208 (2002)

[33] G. G. Batrouni and R. T. Scalettar, Phys. Rev. Lett. 84, 1599 (2000)

[34] F. Hébert, G. G. Batrouni, R. T. Scalettar, G. Schmid, M. Troyer, and A. Dorneich, Phys. Rev. B 65, 014513 (2001).

[35] N. V. Prokof'ev, B. V. Svistunov, and I. S. Tupitsyn, Journal of Experimental and Theoretical Physics 87, 310 (1998)

[36] It has to be stressed that the glassy scenarios that we find at large $V / t$, i.e., where the system is essentially classical, have been obtained by some of us in similar models via molecular dynamics approaches which give access to realtime dynamics [17]. This points out how the appearance of stable disordered states in our work is not an artifact of the adopted QMC algorithm. The same applies for the OOE states at intermediate $V / t$ where our algorithm becomes even more efficient. Indeed, for small $V / t$ our quench protocol is ineffective, and equilibrium is reached even if annealing is not performed. 